Kogan SC. Recurring chromosomal abnormalities in leukemia in PML-RARA transgenic mice identify cooperating events and genetic pathways to acute promyelocytic leukemia. Blood. 2003;102(3):1072-1074.

7. Lallemand-Breitenbach V, Zhu J, Kogan S, Chen Z, de The H. Opinion: how patients have benefited from mouse models of acute promyelocytic leukaemia. Nat Rev Cancer. 2005;5(10):821-827.

8 . Kogan SC. Mouse models of acute promyelocytic leukemia. Curr Top Microbiol Immunol. 2007;313:3-29.

9. Chan IT, et al. Oncogenic K-ras cooperates with PMLRAR alpha to induce an acute promyelocytic leukemia-like disease. Blood. 2006;108(5):1708-1715.
10. Wartman LD, et al. Sequencing a mouse acute promyelocytic leukemia genome reveals genetic events relevant for disease progression. J Clin Invest. 2011; 121(4):1445-1455

11. Ley TJ, et al. DNMT3A mutations in acute myeloid leukemia. NEngl J Med. 2010;363(25):2424-2433.

12. Mardis ER, et al. Recurring mutations found by sequencing an acute myeloid leukemia genome. N Engl J Med. 2009;361(11):1058-1066.

13. Campbell PJ, Green AR. The myeloproliferative disorders. N Engl J Med. 2006;355(23):2452-2466.

14. Mullighan CG, et al. JAK mutations in high-risk childhood acute lymphoblastic leukemia. Proc Natl
Acad Sci U S A. 2009;106(23):9414-9418.

15. Xiang $Z$, et al. Identification of somatic JAK1 mutations in patients with acute myeloid leukemia. Blood. 2008;111(9):4809-4812.

16. Jeong EG, et al. Somatic mutations of JAK1 and JAK3 in acute leukemias and solid cancers. Clin Cancer Res. 2008;14(12):3716-3721.

17. Flex E, et al. Somatically acquired JAK1 mutations in adult acute lymphoblastic leukemia. J Exp Med. 2008;205(4):751-758

18. Constantinescu SN, Girardot M, Pecquet C. Mining for JAK-STAT mutations in cancer. Trends Biochem Sci. 2008;33(3):122-131.

\title{
Dietary nitrate, nitric oxide, and restenosis
}

\author{
John P. Cooke and Yohannes T. Ghebremariam
}

Stanford Cardiovascular Institute, Stanford, California, USA.

\begin{abstract}
Endothelium-derived NO controls the contractility and growth state of the underlying vascular smooth muscle cells and regulates the interaction of the vessel wall with circulating blood elements. Acute injury of the vessel wall denudes the endothelial lining, removing homeostatic regulation and precipitating a wave of events leading to myointimal hyperplasia. In this issue of the JCI, Alef and colleagues provide evidence that in the injured vessel wall, the disruption of the NOS pathway is countered by induction of xanthine oxidoreductase, an enzyme capable of producing NO from nitrite. In addition, they link low dietary nitrite levels to increased severity of myointimal hyperplasia following vessel injury in mice.
\end{abstract}

\section{The role of endothelium-derived NO in vascular homeostasis}

The endothelium is a diaphanous film of tissue comprising a single-cell monolayer that lines the luminal surface of all blood vessels. Despite its fragility, this delicate membrane exerts dramatic control over vascular homeostasis. Endotheliumderived factors control the contractility and growth state of the underlying VSMCs, and regulate the interaction of the vessel wall with circulating blood elements. NO is arguably the most influential of these paracrine substances.

$\mathrm{NO}$ is derived from the metabolism of L-arginine by NOS. NO activates soluble guanylate cyclase to produce cGMP, which participates in signaling pathways involving phosphorylation (1). Furthermore, NO regulates the activities of enzymes and signaling proteins by S-nitrosylation of thiol moieties (2). The endothelial synthesis of this potent molecule is highly regulated, in response to hemodynamic (e.g.,

Conflict of interest: John P. Cooke is an inventor on Stanford University patents related to therapeutic modulation of the NOS pathway.

Citation for this article: J Clin Invest. doi:10.1172/ JCI57193. shear stress) as well as humoral (e.g., serotonin) factors (3). Endothelium-derived NO suppresses platelet adhesion, leukocyte infiltration, and VSMC proliferation and migration (3). Finally, endotheliumderived NO suppresses the endothelial expression of adhesion molecules and chemokines that would otherwise initiate vascular inflammation (3). Cardiovascular risk factors (e.g., hypercholesterolemia, diabetes mellitus) impair the synthesis and bioactivity of endothelium-derived NO $(4,5)$. Their adverse effect on the NOS pathway is mediated by asymmetric dimethylarginine (ADMA; the endogenous antagonist of NO synthesis) and by the vascular generation of superoxide anion $\left(\mathrm{O}_{2}{ }^{-}\right)$, which degrades $\mathrm{NO}$ to peroxynitrite anion $\left(\mathrm{ONOO}^{-}\right)(4,5)$.

\section{Myointimal hyperplasia: response to vascular injury and loss of endothelial homeostasis}

Acute injury of the vessel wall (as occurs with balloon angioplasty) denudes the endothelium, fractures the internal elastic lamina, and damages the underlying VSMCs, removing homeostatic regulation and precipitating a wave of events that can lead to myointimal hyperplasia. Plate- lets adhere to collagen in the damaged vessel wall, and secrete PDGF and other proliferative factors. Infiltrating immune cells secrete inflammatory cytokines that contribute to cellular proliferation, and injured VSMCs release FGF. Vascular progenitor cells circulating in the blood may also contribute to the injury response (6). In the absence of the moderating influence of the endothelium, VSMCs from the media proliferate and migrate into the intimal space, and secrete extracellular matrix. This phenotypic modulation is transcriptionally regulated by CArG box DNA sequences within promoter chromatin of VSMC genes (7).

At the site of vascular injury, arginase is induced, converting arginine to urea and ornithine (8). Ornithine serves as a precursor for polyamines that are known to stimulate VSMC proliferation. Although the endothelial source of $\mathrm{NO}$ generation is lost, the activated VSMCs and the infiltrating immune cells express the inducible form of NOS (iNOS). Unfortunately, this enzyme is an imperfect replacement for endothelial NO synthase (eNOS). Unlike eNOS, which produces small amounts of $\mathrm{NO}$ in a compartmentalized and regulated fashion, iNOS is constitutively active, with a $V_{\max }$ that is a thousand-fold that of eNOS. As a result, iNOS quickly outstrips the reduced supply of arginine and donates electrons to oxygen, generating $\mathrm{O}_{2}^{-}$(9). Superoxide anion combines with $\mathrm{NO}$ to form the cytotoxic radical $\mathrm{ONOO}^{-}$. Subsequent activation of oxidant-sensitive transcriptional pathways increases VSMC proliferation and migration (10), contributing to the myointimal lesion. 


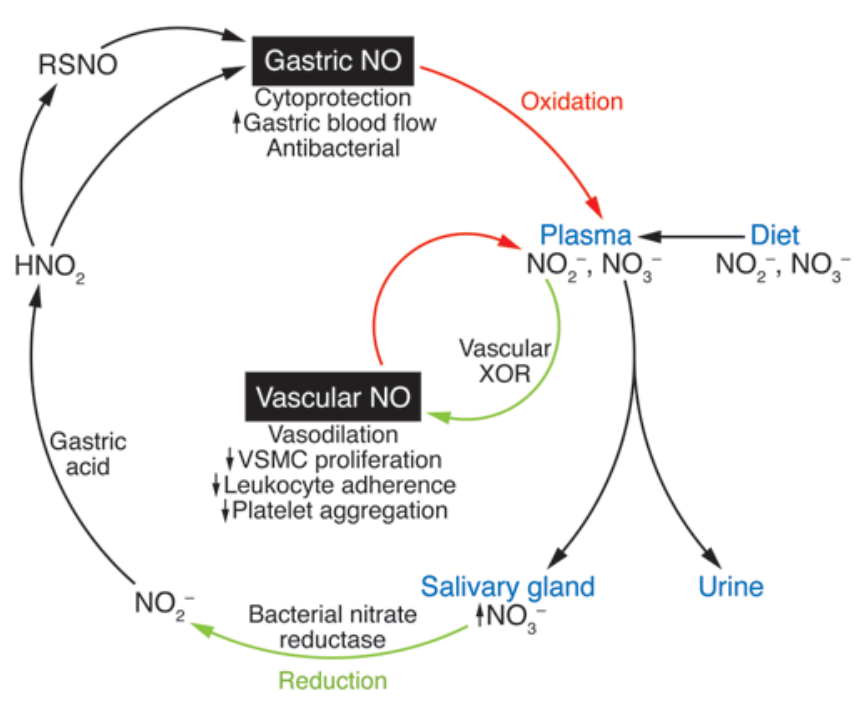

\section{Figure 1}

Dietary nitrite and nitrate are absorbed in the gastrointestinal tract. Plasma nitrate is concentrated in the salivary gland and undergoes reduction by bacteria in the mouth to nitrite. Salivary nitrate is converted in the stomach to nitrous acid, which may undergo spontaneous degradation to NO or which may nitrosylate proteins in the gut mucosa. Gastric NO is protective of the gut mucosa. Plasma nitrite may be converted by XOR in the vessel wall to produce vascular NO, which has homeostatic actions that preserve vessel patency. $\mathrm{HNO}_{2}$, nitrous acid; $\mathrm{NO}_{2}{ }^{-}$, nitrite; $\mathrm{NO}_{3}{ }^{-}$, nitrate; RSNO, S-nitrosothiol-containing peptide or protein.

\section{A new role for exogenous nitrites in restoring vascular homeostasis}

In this issue of the JCI, Alef and colleagues (11) provide evidence that in the injured vessel wall, the disruption of the NOS pathway is countered by induction of xanthine oxidoreductase (XOR). They studied alterations in vascular NOS levels after injury of the rat carotid artery by balloon angioplasty. One week after vascular injury, arginase- 1 expression and activity were increased in the injured vessel wall. They also confirmed that vascular injury induced the expression of vascular iNOS. However, NO biosynthesis (as reflected by vascular nitrosothiols) was not increased, except in the presence of an arginase inhibitor, indicating that L-arginine had become rate limiting for NO production.

In an attempt to correct the deficit in NO biosynthesis, the investigators administered sodium nitrite to the animals before vascular injury. Sodium nitrite reduced myointimal hyperplasia after vascular injury by $50 \%-80 \%$, depending upon the route of administration. This benefit was associated with a 10 -fold increase in NO generation by the injured vessels, an effect that was not attenuated by the NOS inhibitor $N$-nitro-L-arginine methyl ester (L-NAME). Sodium nitrite increased NO generated by cultured VSMCs, also in a NOS-independent manner. Furthermore, sodium nitrite inhibited proliferation of the cells in vitro. This effect was associated with upregulation of the cyclin-dependent kinase inhibitor $\mathrm{p} 21^{\text {Wafl/Cip } 1}$, which is known to mediate in part the antiproliferative effect of NO. In subsequent studies, they documented that regression of estab- lished myointimal hyperplasia could be induced by supplemental sodium nitrite.

How did sodium nitrite lead to the vascular generation of NO? XOR is an enzyme that is capable of producing $\mathrm{NO}$ from nitrite. Alef et al. observed that vascular injury induced the expression and activity of XOR in the vessel wall. They observed that the XOR antagonist allopurinol blocked the antiproliferative action of sodium nitrite in vitro and abrogated the therapeutic effect of dietary nitrite on the injured murine carotid artery. These studies suggest that dietary nitrite was converted to NO by XOR in the vessel wall. A dietary source of nitrite was important in this response, as a reduction in dietary nitrogen oxides aggravated the myointimal hyperplasia after vascular injury. Furthermore, dietary tungsten (which inhibits XOR) also increased the severity of myointimal hyperplasia. These findings are consistent with previous observations that nitrite may be converted to NO in tissues and in blood, particularly in states of hypoxia and acidosis $(12,13)$. Alternatively, or in addition, others have proposed that the bioactivation of nitrite to NO may involve hemoglobin, myoglobin, neuroglobin, aldehyde oxidase, carbonic anhydrase, eNOS, and mitochondrial enzymes, and in particular aldehyde dehydrogenase (14).

\section{Are dietary nitrates and nitrites therapeutic?}

Until very recently, nitrate $\left(\mathrm{NO}_{3}{ }^{-}\right)$and nitrite $\left(\mathrm{NO}_{2}^{-}\right)$were viewed as contaminants in drinking water and in cured meats, and at the very least relatively inactive metabolites of endogenous NO. However, dietary
$\mathrm{NO}_{2}{ }^{-}$and $\mathrm{NO}_{3}{ }^{-}$are enriched in leafy green vegetables, and some have argued that these nitrogen oxides participate in the cardiovascular benefit of a vegetarian or Mediterranean diet (15). This argument is based on emerging support for an endogenous nitrate cycle in humans that has important physiological benefits (Figure 1). The salivary glands extract $\mathrm{NO}_{3}{ }^{-}$from the plasma, concentrating it 10 - to 20 -fold (15). Anaerobic bacteria in the mouth that express nitrate reductases subsequently convert the nitrate to nitrite. When nitrite-rich saliva is swallowed, it reacts with the acidic gastric juices to form nitrous acid $\left(\mathrm{HNO}_{2}\right)$, which is exceptionally active as an antibacterial. Subsequently, $\mathrm{HNO}_{2}$ decomposes into $\mathrm{NO}_{2}{ }^{-}$, $\mathrm{NO}$, and water. Alternatively or in addition, it is possible that the formation of $\mathrm{HNO}_{2}$ (in equilibrium with $\mathrm{N}_{2} \mathrm{O}_{3}$, a potent nitrosating agent), leads to the generation of nitrosothiols. In any event, NO increases local blood flow and protects the gastric mucosa (15).

The current study indicates that a similar process occurs in the vessel wall, where $\mathrm{NO}_{2}^{-}$is also converted to NO. The conversion of nitrite to NO by the enzyme XOR (and/or other metabolic pathways as discussed above) could replace or augment endothelial production of NO and thereby inhibit processes known to contribute to atherosclerosis, restenosis, and vascular inflammation. This human nitrate cycle may account for some of the anti-hypertensive effect observed in diets that are enriched in vegetables and fruit, such as in the DASH (Dietary Approaches to Stop Hypertension) study (16). Consistent with this hypothesis are the observations from small clinical studies that administration of dietary nitrate 
or nitrite can increase plasma cGMP and reduce blood pressure $(15,17,18)$. However, like any potential therapy, there are potential adverse effects $(15,18)$. Dietary supplementation of nitrites can cause methemoglobinemia and interfere with oxygenation of the blood. Furthermore, dietary nitrite and nitrate can contribute to the formation of potentially carcinogenic nitrosamines, although the epidemiological association of dietary nitrogen oxides with cancer has been questioned (19). Accordingly, until there is more guidance from larger, randomized clinical trials, direct supplementation of the diet with nitrite or nitrate salts seems inadvisable. Adherents of the hypothesis may be served best by generous helpings of leafy green vegetables.

\section{Acknowledgments}

The authors thank Jonathan Stamler for comments on this work. This work was supported in part by grants from the NIH (RC2HL103400, 1U01HL100397, and K12 HL087746) and by the Tobacco-Related Disease Research Program of the University of California (18XT-0098).
Address correspondence to: John P. Cooke, Stanford Cardiovascular Institute, 300 Pasteur Drive, A260 MC 5319, Stanford, California 94305, USA. Phone: 650.723.6459; Fax: 650.723.8392; E-mail: john.cooke@ stanford.edu.

1. Kots AY, Martin E, Sharina IG, Murad F. A short history of cGMP, guanylyl cyclases, and cGMPdependent protein kinases. Handb Exp Pharmacol. 2009;191(191):1-14.

2. Lima B, Forrester MT, Hess DT, Stamler JS. S-nitrosylation in cardiovascular signaling. Circ Res. 2010;106(4):633-646.

3. Cooke JP. Flow, NO, and atherogenesis. Proc Natl Acad Sci U S A. 2003;100(3):768-770.

4. Cooke JP. Asymmetrical dimethylarginine: the Uber marker? Circulation. 2004;109(15):1813-1818.

5. Münzel T, Gori T, Bruno RM, Taddei S. Is oxidative stress a therapeutic target in cardiovascular disease? Eur Heart J. 2010;31(22):2741-2748.

6. Patel SD, Waltham M, Wadoodi A, Burnand KG, Smith A. The role of endothelial cells and their progenitors in intimal hyperplasia. Ther $A d v$ Cardiovasc Dis. 2010;4(2):129-141.

7. McDonald OG, Owens GK. Programming smooth muscle plasticity with chromatin dynamics. Circ Res. 2007;100(10):1428-1441.

8. Morris SM Jr. Recent advances in arginine metabolism: roles and regulation of the arginases. $\mathrm{BrJ}$ Pharmacol. 2009;157(6):922-930.

9. Xia Y, Dawson VL, Dawson TM, Snyder SH, Zweier JL. Nitric oxide synthase generates superoxide and nitric oxide in arginine-depleted cells leading to peroxynitrite-mediated cellular injury. Proc Natl Acad Sci U S A. 1996;93(13):6770-6774.

10. Lassègue B, Griendling KK. NADPH oxidases: functions and pathologies in the vasculature. Arterioscler Thromb Vasc Biol. 2010;30(4):653-661.

11. Alef MJ, et al. Nitrite-generated NO circumvents dysregulated arginine/NOS signaling to protect against intimal hyperplasia in Sprague-Dawley rats. J Clin Invest. 2011;121(4):1646-1656.

12. Feelisch $M$, et al. Tissue processing of nitrite in hypoxia: an intricate interplay of nitric oxide-generating and -scavenging systems. J Biol Chem. 2008; 283(49):33927-33934.

13. Zweier JL, Li H, Samouilov A, Liu X. Mechanisms of nitrite reduction to nitric oxide in the heart and vessel wall. Nitric Oxide. 2010;22(2):83-90.

14. Lundberg JO, et al. Nitrate and nitrite in biology, nutrition and therapeutics. Nat Chem Biol. 2009; 5(12):865-869.

15. Weitzberg E, Hezel M, Lunderg JO. Nitratenitrite-nitric oxide pathway: Implications for anesthesiology and intensive care. Anesthesiology. 2010;113(6):1460-1475.

16. Dykhuizen RS, et al. A clinical trial of the effects of dietary patterns on blood pressure. DASH Collaborative Research Group. N Engl J Med. 1997; 336(16):1117-1124

17. Kapil V, et al. Inorganic nitrate supplementation lowers blood pressure in humans: role for nitritederived NO. Hypertension. 2010;56(2):274-281.

18. Tang Y, Jiang H, Bryan NS. Nitrite and nitrate: cardiovascular risk - benefit and metabolic effect. Curr Opin Lipidol. 2011;22(1):11-15.

19. Powlson DS, et al. When does nitrate become a risk for humans? J Environ Qual. 2008;37(2):291-295.

\title{
Neutrophils give us a shock
}

\section{Clifford A. Lowell}

\author{
Department of Laboratory Medicine, UCSF, San Francisco, California, USA.
}

\begin{abstract}
Systemic anaphylaxis is generally recognized as a severe allergic reaction caused by IgE-mediated activation of mast cells, leading to massive release of vasoactive mediators that induce acute hypotension and shock. However, experimental evidence in mice suggests that this view is too simple. Using a variety of techniques to manipulate immune cell makeup, Jönsson et al. come to the conclusion in this issue of the JCI that recognition of IgG1 and IgG2 antibodies by $F c \gamma$ RIII and Fc $\gamma$ RIV receptors on neutrophils is a major pathway for induction of anaphylaxis. These exciting results suggest that we have to reevaluate our models for anaphylaxis in humans, which will have a direct impact on our therapeutic approaches for prevention of this potential deadly hypersensitivity reaction.
\end{abstract}

I turned to Wikipedia when I was searching for a way to explain the basics of anaphylaxis and came across the following statement: "True anaphylaxis is caused by degranulation of mast cells or basophils mediated by immunoglobulin E (IgE)." This is the classic teaching, present in all

Conflict of interest: The author has declared that no conflict of interest exists.

Citation for this article: J Clin Invest. doi:10.1172/ JCI57296. the immunology textbooks, but the paper in this issue of the JCI by Jönsson et al. (1) informs us that this view is, at best, incomplete. Instead, we learn that anaphylaxis can be mediated by neutrophils recognizing IgG/antigen complexes. In addition to turning around our understanding of anaphylaxis, this paper adds to the growing list of neutrophil functions besides just bacterial killing and protease production (2). Lately we have learned that neutrophils are major sourc- es of cytokines and chemokines $(3,4)$. They play a direct role in influencing the recruitment and activation of monocytes/ macrophages, T cells, and NK cells during inflammation (5-7). Neutrophils have been implicated as the primary initiators of immune complex-mediated diseases $(8,9)$. And now the shocking news (pun intended!) that they are major players in initiating anaphylaxis.

\section{The history of anaphylaxis}

Anaphylaxis is an acute, multisystem, severe type I hypersensitivity reaction that develops in minutes to hours following antigen exposure (10). In its mildest forms, it results in rashes (hives), wheezing, and some gastrointestinal symptoms (cramping, bloating). In its more severe forms, patients develop bronchoconstriction with hypoventilation, systemic vasodilation (leading to frank shock), cardiac dysrhythmias, and central nervous system abnormalities. Anaphylaxis most often 